This is the final peer-reviewed accepted manuscript of:

M. Eesaee, E. David, N. R. Demarquette, D. Fabiani and F. Palmieri

"Charge transport and accumulation in clay-containing LDPE nanocomposites"

in IEEE Transactions on Dielectrics and Electrical Insulation, vol. 26, no. 1, pp. 292-299, Feb. 2019

The final published version is available online at:

https://doi.org/10.1109/TDEl.2018.007541

Rights / License:

This article may be used for non-commercial purposes in accordance with Wiley Terms and Conditions for Use of Self-Archived Versions. The terms and conditions for the reuse of this version of the manuscript are specified in the publishing policy. For all terms of use and more information see the publisher's website.

This item was downloaded from IRIS Università di Bologna (https://cris.unibo.it/)

When citing, please refer to the published version. 


\title{
Charge Transport and Accumulation in Clay-containing LDPE Nanocomposites
}

\author{
Mostafa Eesaee, Eric David and Nicole R. Demarquette \\ École de Technologie Supérieure (ETS) \\ Department of Mechanical Engineering \\ 1100 Notre-Dame Street West, Montreal, Quebec, H3C 1K3 Canada
}

\author{
Davide Fabiani and Fabrizio Palmieri \\ University of Bologna \\ Department of Electrical Engineering, \\ Viale Risorgimento 2, 40136 Bologna, Italy
}

\begin{abstract}
This paper discusses the charge transport and accumulation in clay-containing LDPE (Low Density Poly-Ethylene) nanocomposites. LDPE is shown to host charges of both polarities in the form of homo and heterocharge when subjected to high electric fields. Addition of nanoclays has been shown to always increase the high field DC conductivity of the nanocomposites. This is shown to actually work in favor of the ability of the material to prevent the accumulation of space charge by slowly, but persistently, allowing space charges to flow across the insulation wall. However, in severe conditions of a combined high electric field and high temperature, the current flow exceeds a threshold where massive injected charges negatively impact the charge profile and the electric field distribution is heavily distorted.
\end{abstract}

Index Terms - conductivity measurement, space charge, PEA, electric field distortion

\section{$1 \quad 1$ INTRODUCTION}

2 UNDERGROUND and submarine cables are important

3 components in the power grid for the transmission and

4 distribution of electricity and it is of vital importance that they

5 should function reliably for many years. To reach this goal and

6 further improvements in transmission capacity, efforts must take

7 place to improve the insulation layer as the heart of the HV cable.

8 In recent decades polymeric extruded insulation cables have been

9 gained attentions in this regard. In particular, the cross-linked

10 polyethylene insulation cables or XLPE has become the primary

11 cable insulation material for both high voltage $\mathrm{AC}$ and $\mathrm{DC}$

12 applications because of its mechanical flexibility, thermal

13 endurance and excellent dielectric properties [1].

14 Despite all the advantages, XLPE cables can suffer from 15 several problems such as thermal degradation and the lack of

16 recyclability [2]. Those employed for high voltage direct-

17 current (HVDC) applications have also been shown to have

18 the tendency to accumulate space charge, which can lead to

19 failure, especially when encountering polarity reversal

20 operations [3]. Interests have been expressed as to improve the

21 performance of HV cables as the use of XLPE cables grew.

22 Nanometric dielectrics, or nanodielectrics, consisting mainly

Manuscript received on 15 July 2018, in final form xx Month 20yy, accepted xx Month 20yy. Corresponding author: M. Eesaee.
23 of a polymeric matrix and a nanometric reinforcement have 24 been the subject of many researches in recent decades. A great 25 choice of nanoreinforcement is layered silicate fillers, for 26 which many nanocomposites have been produced and 27 analyzed. The incorporation of small percentage of nanoclay 28 has shown to improve the thermal and mechanical properties 29 of polymers [4]. They are also reported to have superior 30 electrical breakdown strength [5], improved partial discharge 31 and volume resistivity to erosion $[6,7]$ but always higher 32 dielectric losses at power frequency [8] comparing to unfilled 33 polymer.

34 In addition, mitigation of space charge accumulation can 35 potentially be reached with such nanocomposites which is of 36 vital importance since less accumulated space charge in the 37 insulation material allows for higher applied electric field at 38 the same cable geometry and as a result the weight and cost of 39 power equipment will be decreased. This can be possible due 40 the barrier effect of the clay platelets, ioning trapping of the 41 inorganic filler and also a slight increase of the DC 42 conductivity allowing space charges to slowly flow across the 43 insulation wall instead of accumulating inside the insulation 44 bulk. Thus, understanding the space charge behavior of clay45 containing polymers is of necessary for designing new 46 materials for HV applications.

47 This manuscript is a continuation to the authors' previous 48 work where it was shown that the addition of clay nanofiller 
1 significantly improves the AC electrical breakdown strength 25 individual high aspect ratio clay platelets were spotted 2 of LDPE, while always results in inferior, but comparable, DC 26 separated from each other and partial exfoliation is achieved 3 electrical breakdown properties [5]. This investigation 27 (Figure 1). The apparent orientation of the nanoparticle was 4 resumes in this report by evaluating the charge transport and 28 related to the high pressure and high temperature condition of 5 accumulation within the same materials to have a general view 29 the molding process of pellets into thin plates. The readers are 6 of their potential for applications as insulating walls in HV 30 referred to the authors' previous work for detailed information 7 cables or for other applications for which surface or internal 31 about the materials, nomenclature and experimental procedure 8 charging is an issue such as spacecraft dielectrics [9]. 32 [5].

\section{EXPERIMENTAL}

\subsection{MATERIAL AND PROCESSING}

11 A premixed LDPE/Clay commercially available masterbatch

12 was diluted with pure LDPE in powder form to achieve clay-

13 containing LDPE nanocomposites with desired concentrations

14 of nanofiller $(1 \%, 2.5 \%, 5 \%, 10 \%$ and $15 \%)$ in a co-rotating

15 twin screw extruder. The masterbatch contains nominal $50 \mathrm{wt}$

$16 \%$ of montmorillonite clay which has been treated with

17 compatibilizing agents to enhance their dispersion within the

18 polymers. The obtained pellets were then press-molded into

19 thin plates with thickness around $280 \mu \mathrm{m}$. Morphology of the

20 as-obtained nanocomposites were investigated in the authors

21 previous work where it was shown in SEM images that stack

22 of nanoclay are uniformly distributed throughout the

23 polyethylene matrix. Further observation through TEM images

24 revealed clear signs of polymer intercalation where numerous
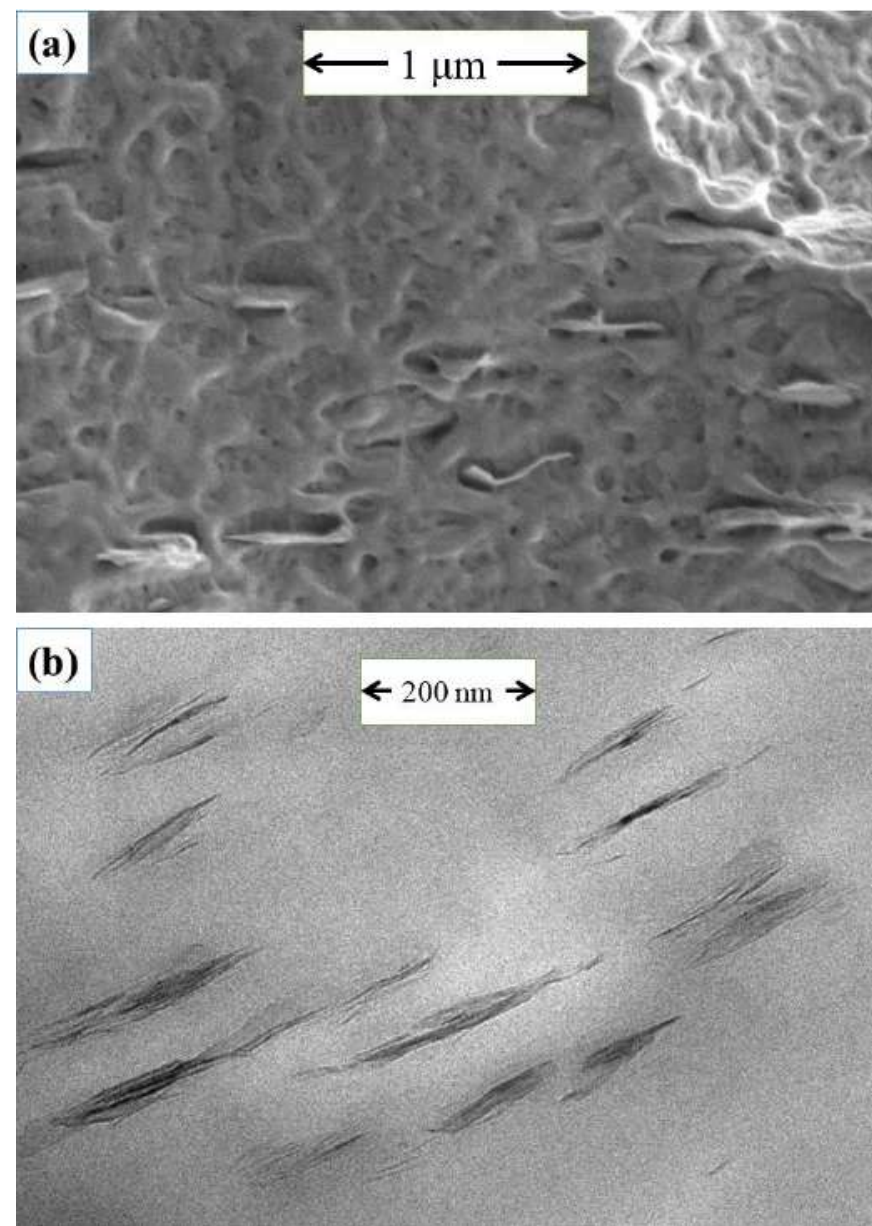

Figure 1. Micrographs of LDPE/5C: a) SEM and b) TEM. (Images are taken from authors' previous work [5])

\subsection{CHARACTERIZATION}

34 Conduction current measurements were conducted using a 35 three electrodes system under a field of $50 \mathrm{kV} / \mathrm{mm}$ at $30{ }^{\circ} \mathrm{C}$. 36 Supplementary measurements have been conducted on neat 37 LDPE and LDPE/2.5C nanocomposite (containing 2.5\% 38 nanoclay) under different fields $(30,40 \& 50 \mathrm{kV} / \mathrm{mm})$ and 39 different temperatures $\left(30,50,70 \& 90{ }^{\circ} \mathrm{C}\right)$ to evaluate the 40 effect of temperature and field variations. Samples were 41 sputtered by gold prior to testing. The system is consisted of 42 main and guard electrodes on one side, and a counter electrode 43 on the other side. The whole system was placed in an oven, 44 where the temperature was stabilized before starting the test. 45 The measurement lasted until the steady state conduction was 46 achieved which took an average time of 24 hours for each 47 sample. An average of the last 100 points after stabilization 48 was considered as the conduction current. Charging and 49 discharging current was continuously monitored using a 50 digital electrometer (Keithley 6514) and the system was 51 computerized using a Labview application.

52 Pulsed Electroacoustic technique (PEA) has been used to 53 measure the space charge profile under the application of DC 54 electric fields. In this method the interaction of a pulsive 55 electric field and space charges inside the sample creates 56 acoustic waves that propagate through the sample to be 57 detected by the transducer. In order to attenuate sonic 58 impedance mismatch, a semiconductive layer was placed 59 between the specimen and HV electrode. While samples were 60 experiencing the DC field for a duration of $10000 \mathrm{~s}$ in the 61 polarization stage, a $\mathrm{HV}$ pulse with an amplitude of $500 \mathrm{~V}$ and 62 duration of $10 \mathrm{~ns}$ was applied to collect the charge profile 63 information within the specimen. At the end of the 64 polarization phase, the HV source was short-circuited to 65 record the depolarization profile for a duration of $3000 \mathrm{~s}$. The 66 space charge measurements have been conducted on different 67 samples at ambient $\left(25{ }^{\circ} \mathrm{C}\right)$ and higher $\left(60{ }^{\circ} \mathrm{C}\right)$ temperature 68 under different electric fields.

\section{RESULTS AND DISCUSSION}

\subsection{ELECTRICAL DC CONDUCTIVITY}

71 High-field DC conductivity measurements are important to 72 gain understanding of conduction mechanisms of insulating 73 materials especially for their usage in HVDC extruded cable 74 systems. Polarization (charging) currents of neat LDPE and its 75 clay-containing nanocomposites over time at $30{ }^{\circ} \mathrm{C}$ and under $7650 \mathrm{kV} / \mathrm{mm}$ electric field are depicted in Figure 2a. The 77 calculated corresponding DC conductivities from the 78 measured conductive current after achieving the steady state 79 are shown in Figure $2 \mathrm{~b}$. For neat LDPE, the polarization 80 current immediately decreases by three decades which 
1 corresponds to the fast polarization. It then gradually 26 observed in the case of CNT-based composites [12] and can 2 decreases over time, corresponding to slow polarization, and 27 also be predicted by Monte Carlo simulation of composites 3 after around $24 \mathrm{~h}$ the steady state is achieved and the current is 28 filled with circular nano-disks [13].

4 believed to be fully conductive. The calculated conductivity 29 On the other hand, reductions in electrical conductivity of 5 for LDPE at $50 \mathrm{kV} / \mathrm{mm}$ is around $1 \times 10^{-15} \mathrm{~S} / \mathrm{m}$ which is close 30 nanocomposites have been reported several times for 6 to the values reported in the literature [10]. Upon addition of 31 nanocomposites containing metal-oxide nanoparticles [14-16]. 7 clay, there is a significant increase in the level of high-field 32 The main explanation given is that during the cooling stage, 8 conductive current and hence the high-field DC conductivity. 33 the polymer molecules are encouraged to regain the natural 9 There is more than 2 decades increase in conductive current 34 polycrystalline morphology where hydrocarbon chains form 10 when only $1 \%$ of clay is incorporated, and it continues to 35 planar crystalline lamellae $10-20 \mathrm{~nm}$ thick and $\sim 100 \mathrm{~nm}$ wide 11 increase towards higher percentages of clay. From 5 to $10 \%$ of 36 growing out from nucleation points to form spherulites. 12 clay loading, there is an increase of almost three decades of 37 Growing crystalline lamella will reject the particles, impurities 13 current and the DC conductivity reaches to $1.9 \times 10^{-12} \mathrm{~S} / \mathrm{m} 38$ and entangled polymer chain structures to form inter-lamella 14 when 15\% of clay is incorporated. The presence of a 39 amorphous phase. In case of nanometric particles, such as 15 maximum value in the time dependence of the current for the 40 metal-oxide, some particles might be left in the inter$16 \mathrm{LDPE} / 1 \mathrm{C}$ nanocomposite is in good agreement with the space- 41 crystalline amorphous phase as crystallization proceeds that 17 charge limited current theory and also in agreement with the 42 will strongly influence the hole/electron tunneling between 18 numerical solution of the transport equations when trapping 43 lamella across the amorphous phase. Due to the difference in 19 and detrapping of charge carriers are taken into account and 44 the band gap of the most metal-oxide nanoparticles with 20 when Schottky conditions are used at the electrodes [11]. It 45 polyethylene, additional potential barriers are involved which 21 also appears that under high electrical field, a sharp increase of 46 results in increased mean tunneling barrier and lowered local 22 the conductivity, similar to a percolation threshold, is already 47 hole inter-lamella transition rate [17]. Consequently, the 23 achieved at a concentration of 1 wt\% (which wasn't observed 48 overall macroscopic mobility and conductivity is lowered. The 24 at lower field). Very low percolation thresholds are typical of 49 number of affected transition depends on the average diameter 25 high aspect ratio inclusions. They are indeed commonly 50 and concentration of the nanoparticle.

(a)
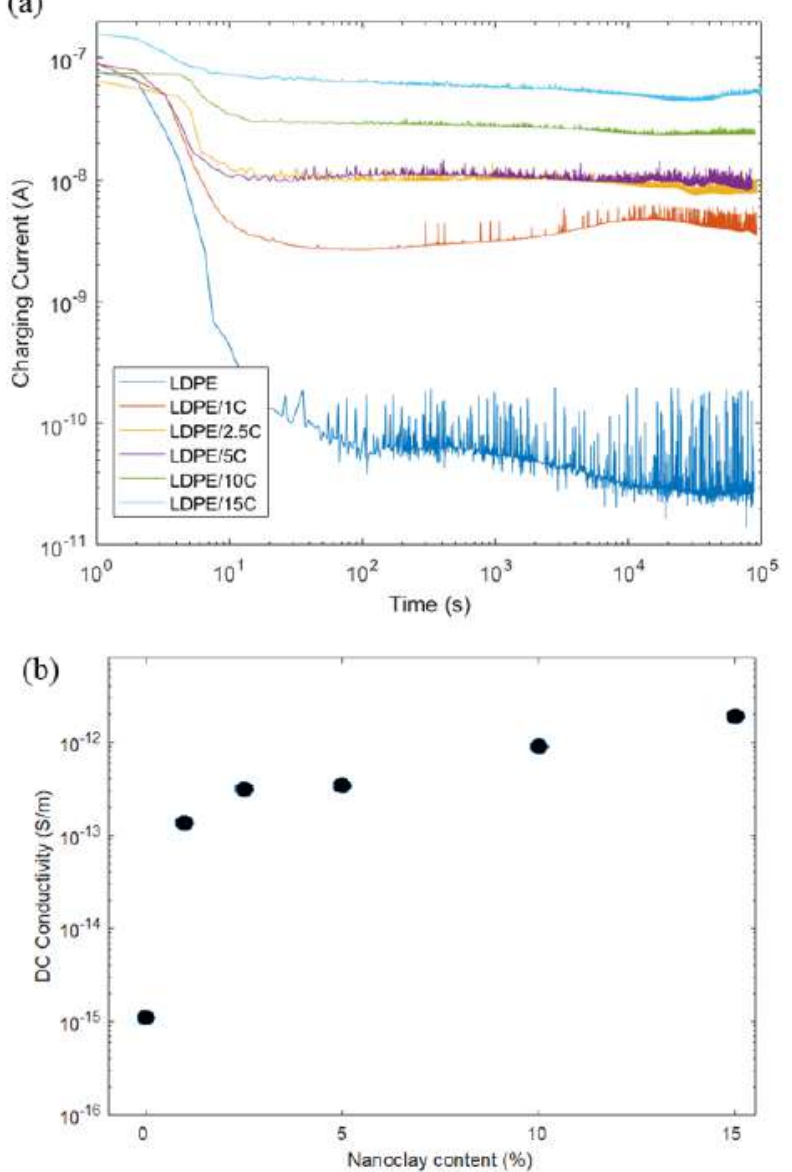

51 In case of layered silicate, such as montmorillonite clay, and 52 due to the high aspect ratio and the tendency to form stacks 53 and agglomerates, they are not expected to be included in the 54 inter-lamella regions. Instead, they are most likely 55 accommodated in the inter-spherulite region and therefore, 56 will not engage directly in a hole or electron tunneling process 57 between crystallites at the nanometric level. The increase in 58 the conductivity of nanocomposites can be more simply 59 related to the formation of high field conductive paths and the 60 increase of mobile charge carriers including free ions 61 introduced by clay nanoparticles due to mobile ion scavenger 62 capability of nesosilicates dispersed within the polymer 63 matrix. It should be noted that the connectivity criterion 64 changes from low field to high field since hopping between 65 inclusions is strongly field-dependant.

66 Since the crystallites do not support ionic transport, the 67 remaining ionic path is in the amorphous phase where the 68 majority of clay nanoparticles are located. The structural 69 symmetry due to the intercalated structure and the huge aspect 70 ratio of clay nanoplatelets even more facilitate the conduction 71 path and make it possible to obtain a massive increase in the 72 conductivity even at low concentration of nanoclay. It should 73 also be noted that even if ionic conductivity is involved in the 74 early conduction process, the long term steady-state current is 75 essentially due to electronic conductivity that takes over after 76 the ionic species reach an equilibrium state resulting in 77 electrode polarization.

78 DC conductivity in polymeric material is temperature and 79 electric field dependent and one way to express this

Figure 2. a) Charging currents of LDPE and its nanocomposites at $30{ }^{\circ} \mathrm{C} 80$ dependency is to use the empirical equation below [18]: and under applied DC electric field of $50 \mathrm{kV} / \mathrm{mm}$; b) calculated DC 81 conductivities from steady state part of the charging currents. 
(1) 22 appears that electric field variation directly influences the 23 conductivity of nanocomposite and a stronger applied field 2 24 enhances the mobility of charge carriers to drift/migrate to the 3 Where $\alpha$ and $\beta$ are the temperature and electric field 24 enhances the mobility of charge carriers to drift/migrate to the
4 coefficients respectively. The dependency to electric field is 26 The dependency to temperature is shown in Figure $3 . \mathrm{b}$ 3 Where $\alpha$ and $\beta$ are the temperature and electric field 25 opposite electrode rather than accumulate at the interface region.
4 coefficients respectively. The dependency to electric field is 26 The dependency to temperature is shown in Figure $3 . \mathrm{b}$ 5 shown in Figure $3 \mathrm{a}$ where the DC conductivities for neat 27 where the DC conductivities of neat LDPE and LDPE/2.5C $6 \mathrm{LDPE}$ and LDPE/2.5C nanocomposite are illustrated at $30^{\circ} \mathrm{C} 28$ nanocomposite are illustrated under $50 \mathrm{kV} / \mathrm{mm}$ of electric 7 and under different fields: 30,40 and $50 \mathrm{kV} / \mathrm{mm}$. The DC 29 field at different temperatures: $30,50,70$ and $90{ }^{\circ} \mathrm{C}$. Applying 8 conductivity for LDPE remains almost the same while it 30 equation (1) fits well to the experimental results if the low 9 monotonously increases for the nanocomposite. According to 31 temperature DC conductivity of the nanocomposite is ignored. 10 equation (1), the estimated electric field coefficients for 32 The temperature coefficients have almost similar values $11 \mathrm{LDPE} / 2.5 \mathrm{C}$ is almost ten times higher than that of neat $\mathrm{LDPE}_{33}\left(\alpha_{2.5 \mathrm{C}}=0.083 \mathrm{~K}^{-1}\right.$, $\left.\alpha_{\mathrm{LDPE}}=0.074 \mathrm{~K}^{-1}\right)$ indicating that temperature $12\left(\beta_{2.5 \mathrm{C}}=0.132 \mathrm{~mm} / \mathrm{kV}, \beta_{\mathrm{LDPE}}=0.014 \mathrm{~mm} / \mathrm{kV}\right)$. Another way to 34 variation effect is mostly controlled by the matrix not by the 13 express the dependency of the DC conductivity towards 35 nanofillers. Generally, it is believed that the conductivity of 14 electric field is the use a power law relationship [19]: 15

16

17

$$
\sigma=\sigma_{0} \exp (\alpha T) E^{\beta}
$$

18 The experimental results fit as well equation (2) with the same 40

19 field coefficient ratio $\left(\beta_{2.5 \mathrm{C}}=5.49, \beta_{\mathrm{LDPE}}=0.55\right)$. More complex 41 36 polymers is thermally activated and Arrhenius-type model can 37 describe this physical phenomenon:

$$
\sigma(T)=\sigma_{0} \exp \left(-E_{a} / k T\right)
$$

20 equations expressing the direct conductivity as a function of both

21 field and temperature can be found in the literature [20].
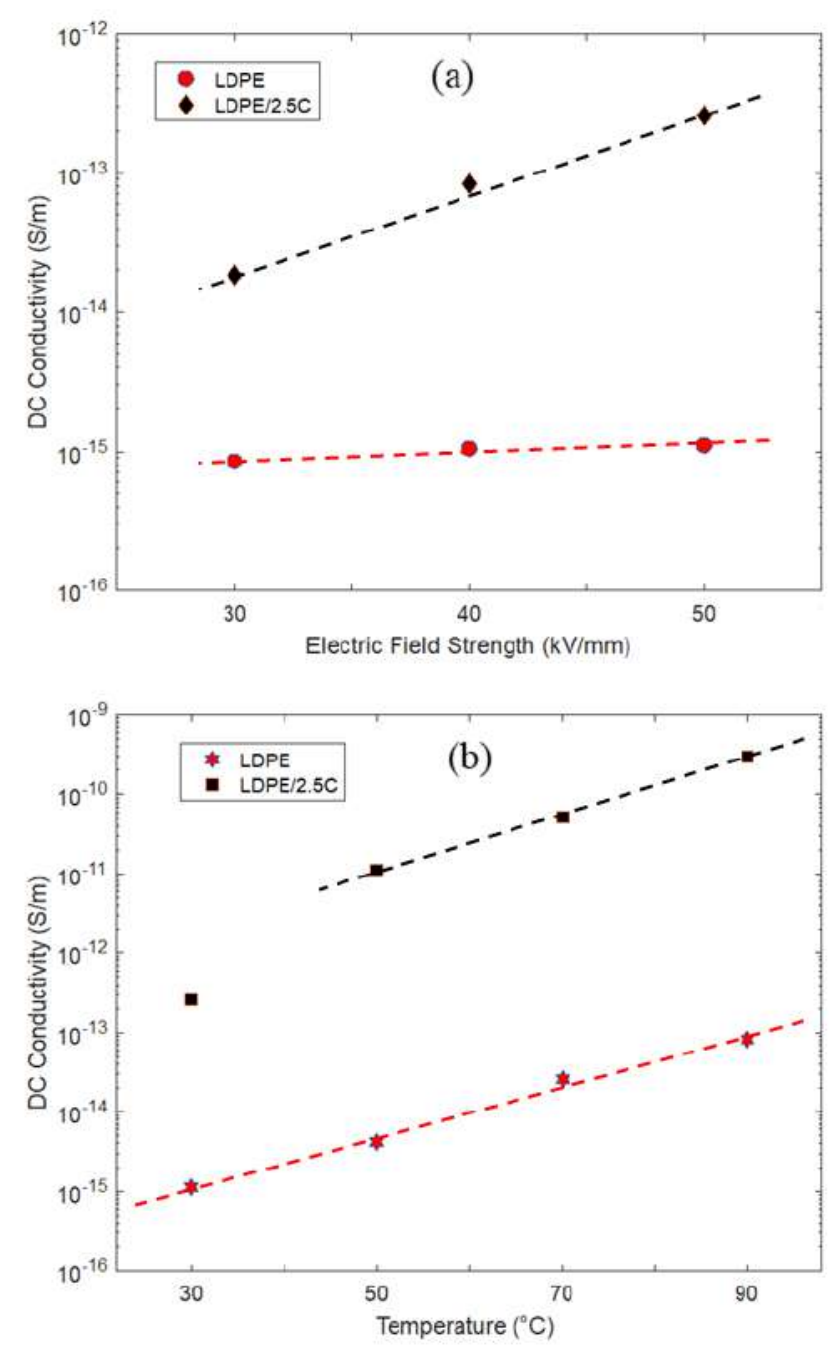
Figure 3. Effect of field variation (a) and Temperature (b) on charging 76 investigated in the following section. currents of LDPE and LDPE/2.5C at $30{ }^{\circ} \mathrm{C}$.
41 where $\sigma_{0}$ represents the pre-exponential factor, $E_{a}$ represents the activation energy and k represents the Boltzmann constant. The estimated activation energy for LDPE/2.5C is slightly 44 higher $(0.84 \mathrm{eV})$ than that of neat LDPE $(0.7 \mathrm{eV})$. However, 45 four points of data are certainly not enough to accurately 46 estimate the activation energy. It was reported in the literature 47 that above $45{ }^{\circ} \mathrm{C}$ the activation energy of LDPE changes from $481 \mathrm{eV}$ to $0.6 \mathrm{eV}$ [21]. This may also explain the low 49 temperature deviation noted for LDPE/2.5C.

50 The increased DC conductivity at higher temperatures 51 following an exponential relation can readily be explained 52 referring to bulk limited conduction mechanisms for which the 53 material is represented by a series of potential wells with 54 given depth and separation. The conduction process then relies 55 on thermally assisted hopping between localized states, which 56 leads, after some approximations, to the exponential 57 expression given in equation (2). Much more detailed (and 58 more sound) models have also been reported using an 59 effective mobility given by an equation similar to (3) and 60 taking also into account bipolar trapping and recombination of 61 carriers as well as Schottky injection at the electrode/dielectric 62 interface [11]. The transport equations can then be numerically 63 solved yield the current density as a function of time. It 64 generally leads, as expected, to an increase of the number of 65 effective charge carriers as well as their mobility resulting in a 66 higher conductivity as the temperature increases.

67 To reach the highest voltage levels and transmission 68 capacity of HVDC power cables the insulating materials must 69 display low DC conductivity, controlled space charge 70 accumulation together with high electrical breakdown 71 strength. The low DC conductivity is favorable but is not the 72 ultimate goal. The major cause of failure in HVDC systems is 73 the accumulated space charge and its distribution is a function 74 of the gradient of the DC conductivity in the insulation layer. 75 The tendency to space charge accumulation is being 
20 Positive charge is accumulated mostly as homocharge close to

2 Figure 4 illustrates the time-dependent charge distribution 21 the anode.

3 profiles of LDPE and LDPE/5C under different poling fields 22 When the electric field is at the highest level $(70 \mathrm{kV} / \mathrm{mm})$, a 4 from $10 \mathrm{kV} / \mathrm{mm}$ to $70 \mathrm{kV} / \mathrm{mm}$ at $30{ }^{\circ} \mathrm{C}$. At the lowest applied 23 big amount of negative charge injected from the $\mathrm{LV}$ electrode 5 electric field $(10 \mathrm{kV} / \mathrm{mm})$ no accumulated space charge is 24 triggers several positive charge packets which transit in the 6 detected in the bulk of LDPE or LDPE/5C nanocomposite. 25 insulation bulk repeatedly during polarization step in a 7 For neat LDPE at $30 \mathrm{kV} / \mathrm{mm}$, a packet of positive charge is 26 background of bulk negative charge. The nanocomposite 8 injected by the HV electrode which gradually penetrates and 27 sample shows the same pattern under $50 \mathrm{kV} / \mathrm{mm}$ of electric 9 disperses in the polymer bulk while a thin layer of negative 28 field, but with a slightly more enhanced negative charge layer 10 homocharge on LV electrode builds up. For nanocomposite, 29 in the middle.

11 however, the increase in the electric field does not appear to 30 Figure 5 plots the maximum field during the polarization for 12 have more than a slight effect on the charge distribution. 31 LDPE and LDPE/5C at four different applied electric fields 13 Indeed, only a very small heterocharge buildup close to the 32 corresponding to the space charge patterns illustrated in the 14 electrodes can be observed at the beginning of depolarization 33 Figure 4 . At $10 \mathrm{kV} / \mathrm{mm}$, the field patterns almost overlap each 15 step. When the electric field goes up to $50 \mathrm{kV} / \mathrm{mm}$, the 34 other, very close to the applied field hinting a probable ohmic 16 positive charge packet leaving the HV electrode is much 35 behavior. For higher applied electric fields, ohmic behavior 17 bigger in LDPE than at $30 \mathrm{kV} / \mathrm{mm}$. The vast amount of 36 does not hold anymore and the maximum fields are always 18 injected positive charge overwhelms negative charge injected 37 above the applied electric fields. The deviation is bigger for 19 from LV electrode, thus spreading in all the insulation bulk. 38 neat LDPE in comparison to LDPE/5C nanocomposite and its

LDPE
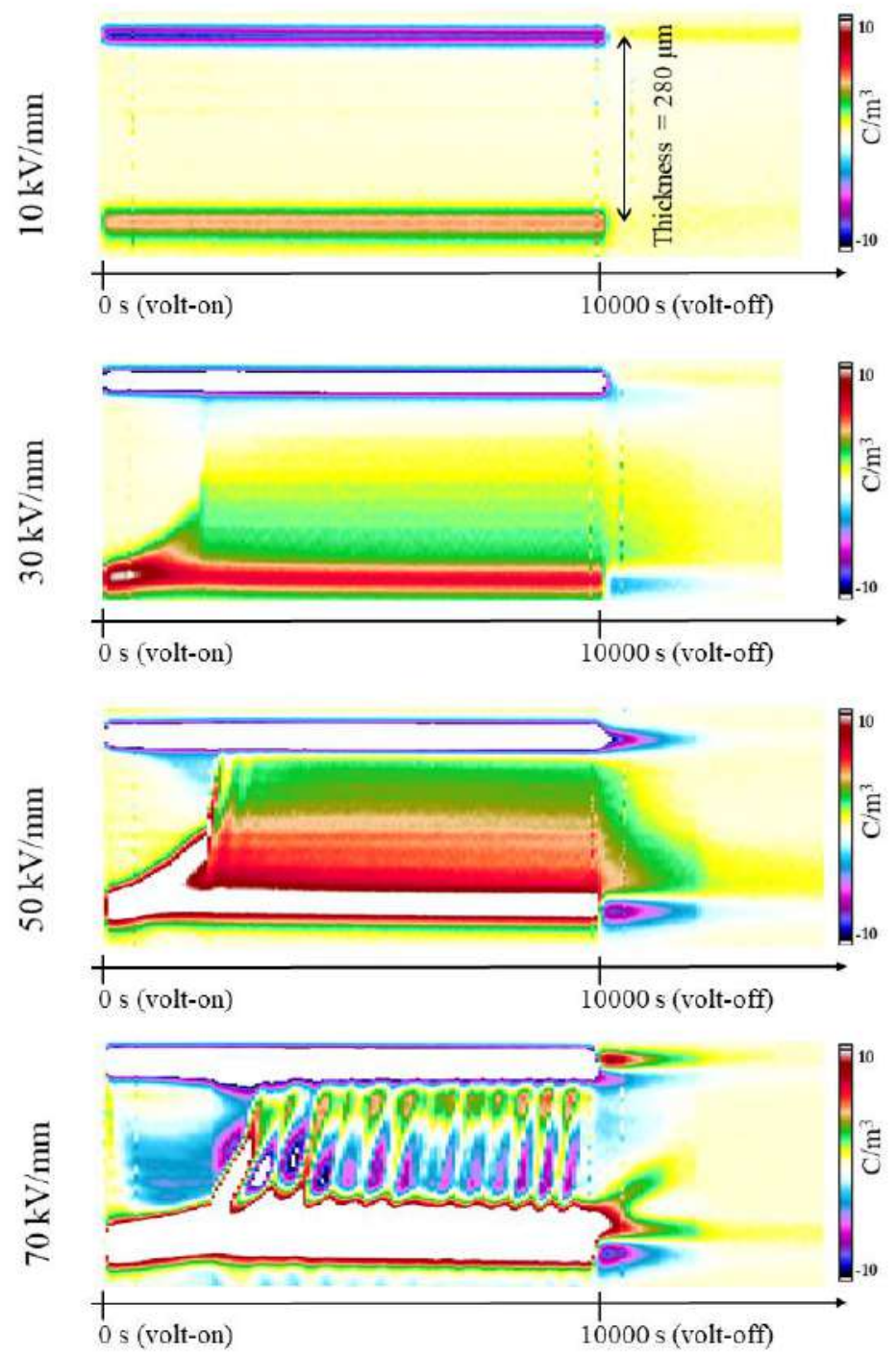

$\mathrm{LDPE} / 5 \mathrm{C}$
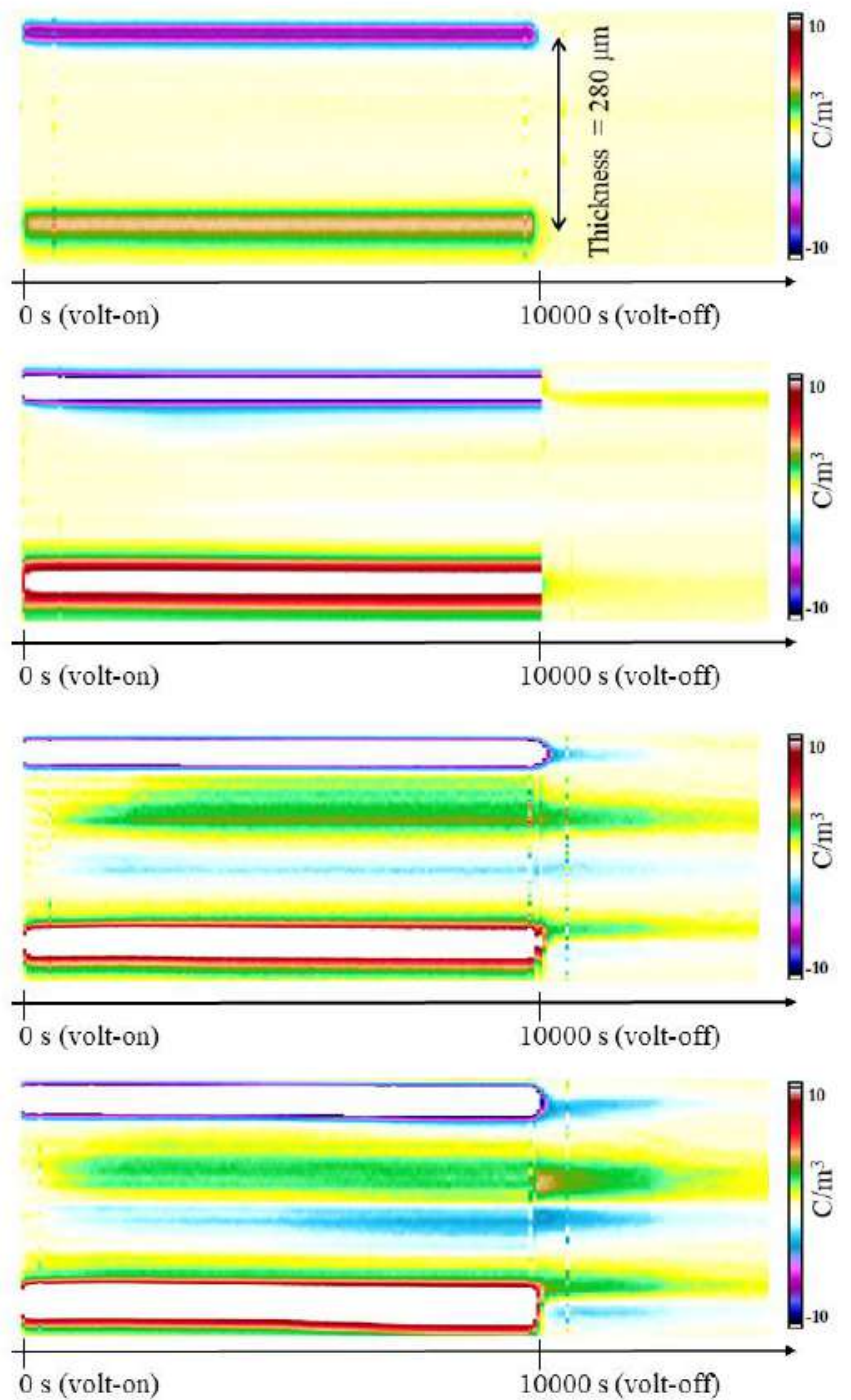

Figure 4. Space charge patterns for LDPE and LDPE/5C nanocomposites at $20{ }^{\circ} \mathrm{C}$ under different applied electric fields. 


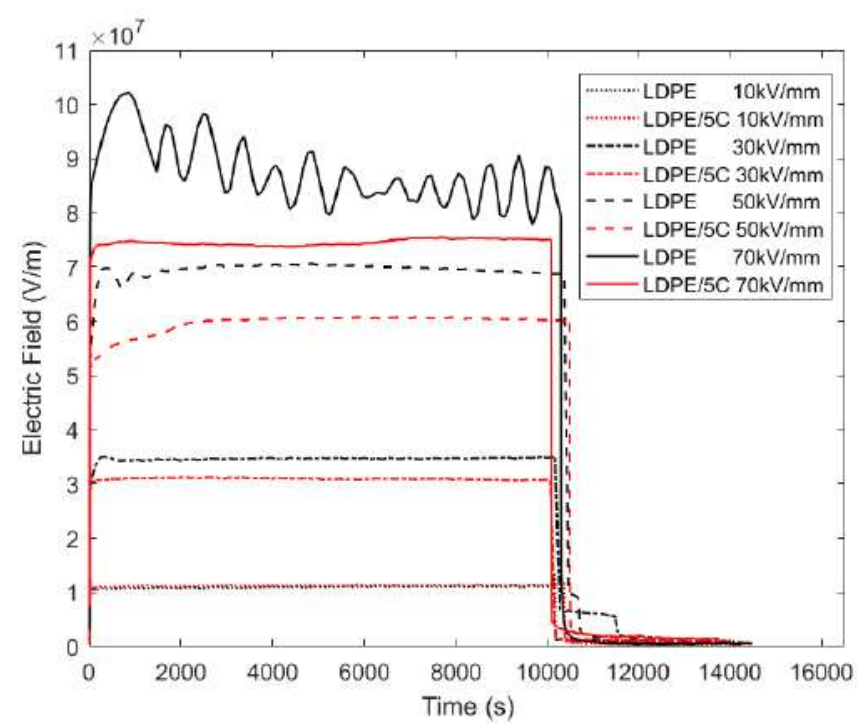

Figure 5. Maximum recorded electric field during the polarization period corresponding to the space charge patterns of Figure 4.

1 intensity increases for stronger applied electric fields at each 2 step. The heterocharge layers at each electrode are responsible 3 for this significant increase. The maximum electric field for $4 \mathrm{LDPE}$ at $70 \mathrm{kV} / \mathrm{mm}$ heavily fluctuates showing a peak every 5 time a charge packet from HV electrode reaches the LV 6 electrode. It occurs, on average, every 700 seconds which can 7 be translated into a charge packet speed of about $4 \times 10^{-7} \mathrm{~m} / \mathrm{s}$ 8 considering the thickness of the LDPE sample $(280 \mu \mathrm{m})$.

9 Attention must be paid when interpreting the space charge 10 accumulation graphs, since at each point the net charge is 11 being displayed. The PEA signal cannot distinguish between 12 the exact amount of positive and negative charge. An area 13 with low charge level could also be due to overlapping of 14 charges having opposite polarity. Therefore, low amount of 15 detected charge in the graphs does not necessary mean that 16 there is low electrical conduction across the thickness of the 17 insulator. In fact, according to the DC conductivity results it 18 was observed that for nanocomposite, even for the lowest 19 loading of nanofiller, the conduction current level is 20 significantly higher than that of neat LDPE.

21 It appears that the increase in the DC conductivity of 22 nanocomposites allows space charge to flow across the 23 insulation wall instead of accumulating inside the insulation 24 bulk. In fact, clay seems to be effective in reducing the space 25 charge not entirely by preventing it from happening but with 26 creating a smooth passage network for charge carriers to be 27 transported away. However, it must be noted that shallow 28 traps are mostly responsible for conduction process, while the 29 incorporation of nanoclay certainly introduces a great portion 30 of deep traps, especially on the surface of nanoparticle [22]. 31 The growth of interfaces of nanoparticles due to the 32 intercalation of LDPE chains increases the density of defects 33 on the surface of nanoparticles resulting in increased number 34 of localized states within the band gap of the material. This 35 will narrow the band gap and hinder the charge transport. As a 36 result, the trap density and trap depth distribution is modified. 37 Overall the presence of nanoclay facilitates the conduction
38 process by introducing more charge carriers to be transported 39 through shallow traps, while suppresses the space charge 40 accumulation by introducing deep traps. This mechanism is 41 particularly efficient at the electrode/insulation interface, 42 because it prevents the injection of excess charge from the 43 electrode at high fields. In other words, most of the charge 44 carriers crossing insulation through shallow traps are extracted 45 at the electrode while some of the electrode-injected charges 46 are immobilized in deep traps near electrodes. This explains 47 the locations of accumulated charges which are near the 48 electrodes in case of nanocomposites, but mostly appears in 49 the bulk of the unfilled LDPE.

50 Space charge patterns for LDPE and its corresponding 51 nanocomposites at elevated temperature $\left(60{ }^{\circ} \mathrm{C}\right)$ under 50 $52 \mathrm{kV} / \mathrm{mm}$ of applied electric fields are illustrated in Figure 6. 53 For neat LDPE a huge amount of negative charge gradually 54 appears in the whole insulation bulk, and no positive charge 55 packet was detected. Surprisingly, despite the massive 56 negative charge injected from LV electrode, it shows 57 relatively lower amount of accumulated charge than the low
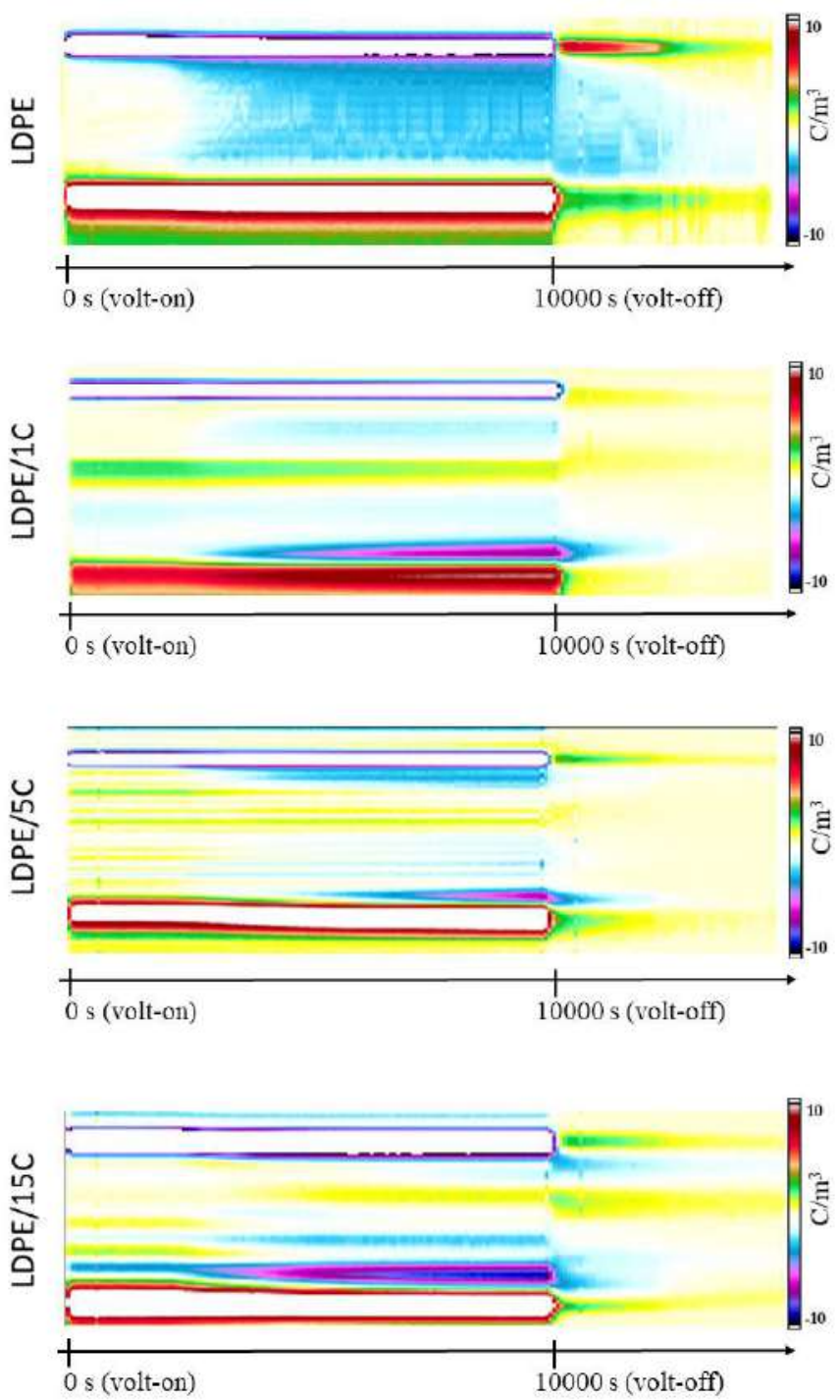

Figure 6. Space charge patterns for LDPE and its clay-containing nanocomposites at $60{ }^{\circ} \mathrm{C}$ and $50 \mathrm{kV} / \mathrm{mm}$ of applied electric field. 

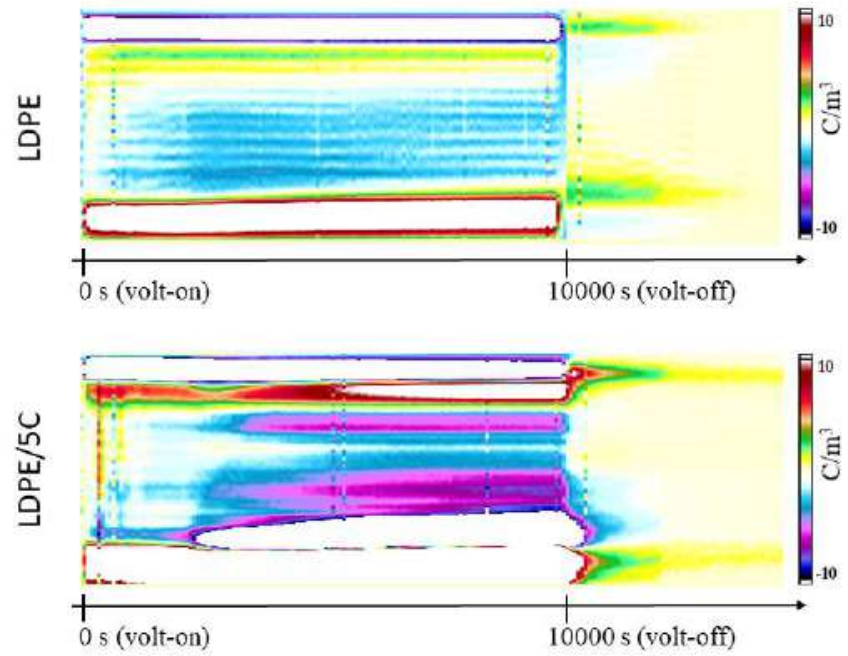

Figure 7. Space charge patterns for LDPE and LDPE/5C at $60{ }^{\circ} \mathrm{C}$ under 70 $\mathrm{kV} / \mathrm{mm}$ of applied electric fields.

1 temperature pattern at the same poling field where injected 41 conditions, the conductivity was found to reach $10^{-10} \mathrm{~S} / \mathrm{m}$, 2 positive charge dominated. The domination of negative charge 42 which is mainly due to electronic conductivity as explained 3 at elevated temperature in LDPE has been recently reported 43 previously. Due to this relatively high value (for a material 4 [23]. Space charge patterns are improved upon incorporation 44 that is mainly non-conductive) significant heterocharge 5 of nanoclay at any concentration: negative heterocharge 45 accumulation occurs close to both electrodes as depicted in 6 gradually builds up near HV electrode while thin layers of 46 Figures 7 and 8 . This fact can be explained again considering 7 positive charge appear in the center. Negative heterocharge 47 the deep traps introduced by nanoclays which prevent 8 build-up instead of dispersing in the whole specimen is 48 injection but also extraction of charges. Since the activation 9 another sign of increased charge mobility (conductivity) where 49 energies for both processes can be different, at high fields / 10 negative charges manage to travel across the thickness to 50 temperatures extraction barrier can prevail, determining a 11 reach HV electrode. The thermal energy provided accelerates 51 significant delay for charge extraction which leads to a huge 12 the detrapping process, combined with the electric field effect 52 heretocharge accumulation.

13 induced by the trapped charge itself that allows a part of 53

14 trapped charges to become mobile and reach the opposite 53

15 electrode.

54 Charge transport and accumulation under high electric fields 16 Space charge patterns for LDPE and LDPE/5C at elevated 55 were studied for neat LDPE and its clay-containing 17 temperature $\left(60^{\circ} \mathrm{C}\right)$ under $70 \mathrm{kV} / \mathrm{mm}$ of applied electric fields 56 nanocomposites. The incorporation of nanoclay in LDPE was 18 are illustrated in Figure 7. For LDPE thin layers of 57 shown to significantly increase the DC conductivity. This 19 heterocharge are close to both electrodes while negative 58 increase was shown to be directly related to the temperature, 20 charges are spread across the thickness of the specimen. The 59 electric field and the loading of nanofiller. The increased 21 nanocomposite sample (LDPE/5C), however, exhibits the 60 conductivity of the nanocomposite with regard to the neat 22 most distorted space charge behavior of all where61 LDPE together with the trapping effect of clay nanoparticles 23 heterocharge layers being immediately built up close to both 62 was found to lead to an improvement in the mitigation process 24 electrodes. The corresponding charge profile for LDPE/5C is 63 of space charge at high fields and room temperature where 25 illustrated in Figure 8 for further analysis. Charge profile 64 extremely low accumulation of space charge was detected for 26 within the specimen is hugely affected by these heterocharge 65 nanocomposites comparing to the base polymer. However, at 27 layers and never reaches the steady state. While the maximum 66 elevated temperature the conductivity of the nanocomposites 28 electric field for LDPE never exceeds $81 \mathrm{kV} / \mathrm{mm}$, for 67 reach a point $\left(\sim 10^{-10} \mathrm{~S} / \mathrm{m}\right)$ where it doesn't provide anymore $29 \mathrm{LDPE} / 5 \mathrm{C}$ it constantly increases and reaches $180 \mathrm{kV} / \mathrm{mm}$ at 68 benefit allowing to leak out the space charge but rather 30 the end of the polarization (not shown here).

69 enhance the space charge accumulation resulting in heavy

31 When combined by high poling field, elevated temperature 70 charge build up close to the electrodes.

32 completely reverses the charge profile trend for neat LDPE

33 and its clay-containing nanocomposite. First for neat LDPE, 71

\section{ACKNOWLEDGMENT}

34 charge packet travelling across the sample were not observed 72 The main sponsor of this project is the Natural Sciences and 35 anymore unlike what was measure at room temperature, as 73 Engineering Research Council of Canada (NSERC). The 36 illustrated in Fig. 4. It seems that the increase of conductivity 74 Authors acknowledge the sincere cooperation of the staff of 37 is high enough to allow disperion of the charge packets and 75 the Laboratory of Innovation Technologies (LIT) at University 38 injected charges to be drifted away but not high enough for 76 of Bologna. 
2 [1] G. Chen, M. Hao, Z. Xu, A. Vaughan, J. Cao, and H. Wang, "Review of 76 high voltage direct current cables," CSEE J. Power and Energy Systems, 77 vol. 1, pp. 9-21, 2015.

[23] Y. Wang, J. Wu, and Y. Yin, "Space charge behavior in low density polyethylene at low temperatures," IEEE Trans. Dielectr. Electr. Insul., 24, pp. 3860-3868, 2017.

5 [2] K. Wu, Y. Wang, X. Wang, M. Fu, and S. Hou, "Effect of space charge

6 in the aging law of cross-linked polyethylene materials for high voltage 79 DC cables," IEEE Electr. Insul. Mag., vol. 33, pp. 53-59, 2017.

8 [3] S. Wang, S. Luo, Y. Tu, C. Wang, and S. Qin, "Effect of polarity 9 reversal on space charge properties of CB/LDPE composite under DC 10 field," IEEE Trans. Dielectr. Electr. Insul., vol. 24, pp. 1349-1354, 2017.

11 [4] S. Pavlidou and C. Papaspyrides, "A review on polymer-layered silicate nanocomposites," Progress in polymer science, vol. 33, pp. 1119-1198, 2008.

14 [5] M. Eesaee, E. David, N. R. Demarquette, and D. Fabiani, "Electrical

15 Breakdown Properties of Clay-Based LDPE Blends and 88

16 Nanocomposites," J. Nanomaterials, vol. 2018, p. 17, 2018.

17 [6] M. Kozako, N. Fuse, Y. Ohki, T. Okamoto, and T. Tanaka, "Surface

18 degradation of polyamide nanocomposites caused by partial discharges

19 using IEC (b) electrodes," IEEE Transactions on Dielectrics and

20 Electrical Insulation, vol. 11, pp. 833-839, 2004.

21 [7] T. Tanaka, "Interface properties and surface erosion resistance," in Dielectric Polymer Nanocomposites, ed: Springer, pp. 229-258, 2010.

23 [8] E. David, M. Fréchette, B. Zazoum, C. Daran-Daneau, A. D. Ngô, and 24 H. Couderc, "Dielectric properties of PE/clay nanocomposites," Journal 25 of Nanomaterials, vol. 2013, p. 65, 2013.

26 [9] A. Hands and K. Ryden, "Experimental Measurement of Low-Intensity 99 78 79

\section{BIOGRAPHIES}

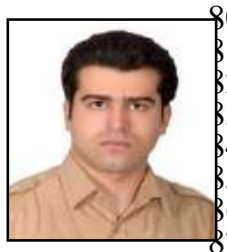

80 Mostafa Eesaee was born in Fariman, Iran, in 1988. He 31 received the B.Sc. degree in Chemical Engineering from 32 Amirkabir University, Tehran, Iran, in 2010, and the 33 M.Sc. degree in Polymer engineering from Sharif 34 University of Technology, Tehran, Iran in 2012. He is a $35 \mathrm{Ph} . \mathrm{D}$. student at École de Technologie Supérieure (ETS) 36 in Montreal, Canada. His research interests include 87 fabrication and characterization of polymeric blends and nanocomposites with a view towards electrical applications. 89 28 Science, vol. 45, pp. 1938-1946, 2017.

29 [10] M. G. Andersson, J. Hynynen, M. R. Andersson, V. Englund, P.-O102 Transactions on Dielectrics and Electrical Insulation.

30 Hagstrand, T. Gkourmpis, et al., "Highly Insulating Polyethylene Blends 31 for High-Voltage Direct-Current Power Cables," ACS Macro Letters, 32 vol. 6, pp. 78-82, 2017

33 [11] S. LeRoy, P. Segur, G. Teyssedre, and C. Laurent, "Description of bipolar charge transport in polythylene using fluid model with a constant mobility: model prediction,” J. Phys. D: Appl. Phys., vol. 37, pp. 298305, 2004.

37 [12] C.A.S. De Lima, L.G. Amurin, N.R. Demarquette, E. David, "Morphological and Electric Properties of Block Copolymer/Carbon

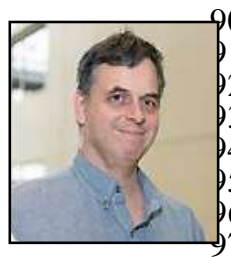

90 Eric David (M'03, SM'07) was born in Montreal in 1 1965. He has been a member of the IEEE since 2002 . $2 \mathrm{He}$ received an M.Sc.A and a Ph.D. in Engineering 3 Physics from the École Polytechnique de Montreal in 941989 and 1996, respectively. He joined the Hydro35 Quebec Research Institute (IREQ) in 1998, and from 362001 to 2002, where he was active in the field of 7 dielectric materials used for underground cables and 98 rotating machines. He is now a professor at the Department of Mechanical Nanotubes Nanocomposites Obtained by Different Methods," 201q Electrical Insulation Conference (EIC), pp. 588-591, 2016.

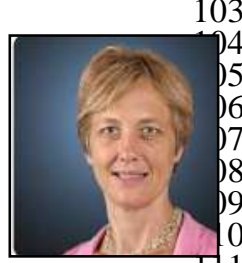

03

4 Nicole Raymonde Demarquette was born in France in 5 1966. She received her B.Sc. from Institute 6 Polytechnique de Grenoble, France in 1989 as well as a 7 Diplome d'Etudes Approfondies in Chemical 8 Engineering from the same institute. She received a 9 M.Sc. and a Ph.D. from McGill University in 1991 and 0 1994, respectively. She also obtained a Livre-docence in

1 Materials Engineering from the University of Sao Paulo, Q12 Brazil. She was a Professor at the Materials Engineering Department of the

113 University of Sao Paulo, Brazil, between 1996 and 2012 and joined École de 41 [13] A.B. Oskouyi, P. Mertiny, "Monte Carlo model for the study of 14 Technologie Supérieure in Montreal in 2012. Her research interests include percolation thresholds in composites filled with circular conductiva 15 the development of new materials based on thermoplastics, rheology, interface

43 nano-disks," Procedia Engineering, vol. 10, pp. 403-408, 2011. 116 science and electrospinning. She is the authors of more than 100 Journal
44 [14] Y. Murakami, M. Nemoto, S. Okuzumi, S. Masuda, M. Nagao, N117 papers. She is an active member of the Society of Rheology, Society of Plastic Hozumi, et al., "DC conduction and electrical breakdown of MgO/LDP耳18 Engineers and Polymer Processing Society. nanocomposite," IEEE Transactions on Dielectrics and Electrical19 Insulation, vol. 15, pp. 33-39, 2008.

48 [15] R. Fleming, A. Ammala, P. Casey, and S. Lang, "Conductivity and space 49 charge in LDPE containing nano-and micro-sized $\mathrm{ZnO}$ particles," IEEE

50 Transactions on Dielectrics and Electrical Insulation, vol. 15, pp. 11851126,2008 .

52 [16] R. Fleming, T. Pawlowski, A. Ammala, P. Casey, and K. Lawrence, "Electrical conductivity and space charge in LDPE containing TiO/sub 2/nanoparticles," IEEE transactions on dielectrics and electrical insulation, vol. 12, pp. 745-753, 2005.

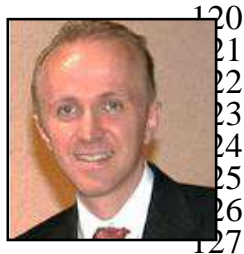

20 Davide Fabiani (M'98) was born in Forli, Italy, in 1 1972. He received the M.Sc. (honors) and Ph.D. degrees 2 in electrical engineering from the University of Bologna 3 in 1997 and 2002, respectively. $\mathrm{He}$ is an associate 24 professor at the Department of Electrical Electronics 5 and Information Engineering of the University of 6 Bologna since 2014. His research interests deal with the 7 effects of voltage distortion on accelerating insulation 128 degradation, characterization of insulating, magnetic, superconducting, 56 [17] T. Lewis, "Charge transport in polyethylene nano dielectrics," IEEH 29 nanocomposites and electret materials, aging investigation and diagnosis of 57 Trans. Dielectr. Electr. Insul., vol. 21, pp. 497-502, $2014 . \quad 130$ power system insulation and, particularly, motor windings subjected to fast 58 [18] Y. Murata, Y. Sekiguchi, Y. Inoue, and M. Kanaoka, "Investigation of 31 repetitive pulses. He is author or co-authors of about 180 papers. He is a electrical phenomena of inorganic-filler/LDPE nanocomposite material,'132 senior member of IEEE, Power Engineering Society (PES), Dielectrics and

601 IEEE Symp. Electr. Materials (ISEIM), pp. 650-653, 2005. 133 Electrical Insulation Society (DEIS) and AEI from 1998. He is a Senior properties of thermo-plastic polyolefin materials," Аnпи. Rep. Confl 35 Inulation. Electr. Insul. Dielectr. Phenom. (CEIDP), pp. 470-473, 2016.

64 [20] Z. Li, B. Du, "Polymeric Insulation for HVDC Estruded Cables:

65 Challenges and Development Directions", Electrical Insulation 66 Magqazine, 2018

67 [21] L. Boudou, V. Griseri, J. Guastavino, and L. Dissado, "Effect of 68 temperature on space charge formation in low density polyethylene-role 69 of antioxidant," IEEE Int. Conf. Solid Dielectr. (ICSD), pp. 252-255, 702004 71 [22] G. C. Montanari, D. Fabiani, F. Palmieri, D. Kaempfer, R. Thomann, of EVA and PP insulation through nanostructure by organophiliq46 measurements and life tests. 137 Fabrizio Palmieri was born in Vergato (Bologna) in
38 1970. He received the Dr. Eng. Degree in electrical
39 engineering from the University of Bologna in 1999 for
10 a thesis on the PEA system. From 2001 to 2003 he was
11 in charge of the testing service for TechImp S.R.I. Since
12 2003 he has been working in the Department of
-3 Electrical Engineering of the University of Bologna. In
44 particular, his interests are in electrical characterization 137 Fabrizio Palmieri was born in Vergato (Bologna) in
38 1970. He received the Dr. Eng. Degree in electrical
39 engineering from the University of Bologna in 1999 for
10 a thesis on the PEA system. From 2001 to 2003 he was
11 in charge of the testing service for TechImp S.R.I. Since
12 2003 he has been working in the Department of
-3 Electrical Engineering of the University of Bologna. In
44 particular, his interests are in electrical characterization 137 Fabrizio Palmieri was born in Vergato (Bologna) in
38 1970. He received the Dr. Eng. Degree in electrical
39 engineering from the University of Bologna in 1999 for
10 a thesis on the PEA system. From 2001 to 2003 he was
11 in charge of the testing service for TechImp S.R.I. Since
12 2003 he has been working in the Department of
-3 Electrical Engineering of the University of Bologna. In
44 particular, his interests are in electrical characterization 137 Fabrizio Palmieri was born in Vergato (Bologna) in
38 1970. He received the Dr. Eng. Degree in electrical
39 engineering from the University of Bologna in 1999 for
10 a thesis on the PEA system. From 2001 to 2003 he was
11 in charge of the testing service for TechImp S.R.I. Since
12 2003 he has been working in the Department of
-3 Electrical Engineering of the University of Bologna. In
44 particular, his interests are in electrical characterization 137 Fabrizio Palmieri was born in Vergato (Bologna) in
38 1970. He received the Dr. Eng. Degree in electrical
39 engineering from the University of Bologna in 1999 for
10 a thesis on the PEA system. From 2001 to 2003 he was
11 in charge of the testing service for TechImp S.R.I. Since
12 2003 he has been working in the Department of
-3 Electrical Engineering of the University of Bologna. In
44 particular, his interests are in electrical characterization 137 Fabrizio Palmieri was born in Vergato (Bologna) in
38 1970. He received the Dr. Eng. Degree in electrical
39 engineering from the University of Bologna in 1999 for
10 a thesis on the PEA system. From 2001 to 2003 he was
11 in charge of the testing service for TechImp S.R.I. Since
12 2003 he has been working in the Department of
-3 Electrical Engineering of the University of Bologna. In
44 particular, his interests are in electrical characterization 137 Fabrizio Palmieri was born in Vergato (Bologna) in
38 1970. He received the Dr. Eng. Degree in electrical
39 engineering from the University of Bologna in 1999 for
10 a thesis on the PEA system. From 2001 to 2003 he was
11 in charge of the testing service for TechImp S.R.I. Since
12 2003 he has been working in the Department of
-3 Electrical Engineering of the University of Bologna. In
44 particular, his interests are in electrical characterization 137 Fabrizio Palmieri was born in Vergato (Bologna) in
38 1970. He received the Dr. Eng. Degree in electrical
39 engineering from the University of Bologna in 1999 for
10 a thesis on the PEA system. From 2001 to 2003 he was
11 in charge of the testing service for TechImp S.R.I. Since
12 2003 he has been working in the Department of
-3 Electrical Engineering of the University of Bologna. In
44 particular, his interests are in electrical characterization 